\title{
Gliese 710 will pass the Sun even closer
}

\section{Close approach parameters recalculated based on the first Gaia data release}

\author{
Filip Berski and Piotr A. Dybczyński
}

\author{
Astronomical Observatory Institute, Faculty of Physics, A. Mickiewicz University, Słoneczna 36, 60-286 Poznań, Poland \\ e-mail: filip.berski@amu.edu.pl
}

Received 3 October 2016 / Accepted 25 October 2016

\begin{abstract}
Context. First results based on Gaia data show that the well-known star Gliese 710 will be the closest flyby star in the next several Myrs and its minimum distance from the Sun will be almost five times smaller than that suggested by pre-Gaia solution.

Aims. The aim of this work is to investigate the proximity parameters and the influence of the close approach of Gliese 710 on the basis of Gaia DR1. Furthermore, we compare new results with previous works based on HIP2 and Tycho 2 catalogues to demonstrate how Gaia improves the accuracy of determination of such phenomena.

Methods. Using a numerical integration in an axisymmetric Galactic model, we determine new parameters of the close encounter for Gliese 710. Adding ten thousand clones drawn with the use of a covariance matrix, we estimate the most probable position and velocity of this star at the minimum distance from the Sun.

Results. Our calculations show that Gliese 710 will pass 13365 AU from the Sun in 1.35 Myr from now. At this proximity it will have the brightness of -2.7 mag and a total proper motion of 52.28 arcsec per year. After the passage of Gliese 710 we will observe a large flux of new long-period comets. Thanks to the Gaia mission, the uncertainties of the minimum distance and time of the close approach are several times smaller than suggested by previous works based on data from earlier observations.
\end{abstract}

Key words. stars: individual: GJ 710 - stars: kinematics and dynamics - solar neighborhood - Oort Cloud

\section{Introduction}

The Gaia mission is a big step in increasing knowledge of our surroundings in the Universe. This mission, launched on 19th December 2013, will result in a map of one billion stars in our part of the Milky Way galaxy. We will know the positions and velocities of these objects and thanks to that, we will be able to study the future and past spatial motions of stars in the solar neighbourhood. This knowledge will be invaluable for research on the origin of long-period comets (LPCs). When Oort formulated his hypothesis about a cloud of cometesimals surrounding the solar system (Oort 1950), he identified close stellar encounters as the only source of observable LPCs. Now we know other mechanisms delivering LPCs to the inner part of the solar system, such as Galactic perturbations (see for example Dybczyński 2005; Kaib et al. 2009), but stellar encounters are still one of the most important and most discussed. Our previous results (Dybczyński \& Berski 2015, hereafter DB15) suggested that the accuracy of determination of close stellar encounters should increase drastically due to Gaia results, and this work serves as a good example.

On September 14th, Data Processing Consortium Executive (DPACE) published the first Gaia catalogue (Gaia DR1, Gaia Collaboration 2016a) based on observations collected between 25 July 2014 and 16 September 2015. One of the components of this catalogue is the Tycho Gaia Astrometric Solution (TGAS, Michalik et al. 2015). TGAS contains five astrometric parameters $\left(\alpha, \delta, \mu_{\alpha *}, \mu_{\delta}\right.$ and $\pi$, with their uncertainties and covariance matrices) for more than two million objects. It contains 1963415 stars from Tycho 2 catalogue (Høg et al. 2000) and 93635 objects from HIPPARcos 2 catalogue (van Leeuwen 2007).
One of the recognised future visitors of the solar system vicinity is Gliese 710. This object was studied in all previous works on this topic, see for example Mullari \& Orlov (1996), Dybczyński \& Kankiewicz (1999), García-Sánchez et al. (2001), Dybczyński (2006). It is a K-dwarf with an estimated mass of 0.61 Solar masses, and its influence on LPCs orbits has been tested by many authors (Dybczyński \& Królikowska 2015; Feng \& Bailer-Jones 2015). Based on pre-Gaia data, its minimum distance from the Sun was estimated to be approximately 59817 AU (Dybczyński \& Berski 2015; Bailer-Jones 2014; Jiménez-Torres et al. 2011; García-Sánchez et al. 2001). Now, with the new data this distance shrinks to only approximately $13 \mathrm{kAU}$, making it the closest approaching star to our Sun within 10-20 Myr of the present day.

In the following section we present the method used to determine the minimum distance and the time of the closest approach, as well as how the accuracy of this solution was estimated. In Sect. 3 we describe the results of our calculations and the comparison with previous results, based mostly on HIP 2 catalogue. In Sect. 4 we discuss the probable effects of this event, and in the last section we present some conclusions.

\section{Methodology}

In this section we present, in general, the methods used to determine new parameters of close stellar approaches. The details of the numerical integration, that is, the frame of reference and equations of motion used, are described in DB15 (Sects. 2.1 and 2.3). In Sect. 2.2 of quoted work we describe in detail the process of drawing a stellar clone. 
The first task after the publication of Gaia DR1 was to find stars approaching the Sun at distances closer than 2 pc. We performed this in a few steps, as follows:

The first step was to find the minimum distance within the rectilinear motion approximation, obtained from simple analytical formulas. To do this, we needed five astrometric parameters and radial velocities; but radial velocities from the Gaia mission will be published in the second release. Instead of searching radial velocities for all Gaia stars, we filtered out 99 per cent of them by assuming some artificial but large velocity of $100 \mathrm{~km} \mathrm{~s}^{-1}$. This assumption follows from statistics: most stars have much smaller radial velocity, and all close approaching stars with that velocity would have much smaller minimum distance than in reality. Since we are interested in both past and future proximities, the sign of this assumed radial velocity is not important. Additionally, to make sure that we did not miss out any stars, we considered all stars with minimum distance $\left(D_{1}\right)$ smaller than $10 \mathrm{pc}$ in the following step. After this first step we obtained approximately 26 thousand candidate stars from more than two million objects in TGAS.

Then we searched for radial velocities (RV) of all the objects with $D_{1}<10 \mathrm{pc}$ in the SIMBAD database ${ }^{1}$ by means of our own software designed specifically for this purpose. If RV was not found for an object, it was removed from the list of potentially close approaching objects. Over six thousand radial velocities for candidate stars were found.

In the last step we numerically integrated the motion of the Sun and interesting objects in an axisymmetric gravitational potential model of the Galaxy taken from Irrgang et al. (2013; see DB15 for details). Then we calculated the motions of only those objects with the true values of RV and with $D_{1}$ smaller than $10 \mathrm{pc}$, without applying any criteria based on time, but typically we followed a star motion for few million years. As a result of this step we obtained a list of 34 stars, approaching and passing the Sun at a distance closer than $2 \mathrm{pc}$. At the top of this list we found Gliese 710, with the minimum distance obtained from numerical integration $D_{\min }=13366 \mathrm{AU}$ in 1.3538 million years from now.

To determine the accuracy of our results we cloned the star ten thousand times using the covariance matrix for five kinematic parameters $\left(\alpha, \delta, \mu_{\alpha^{*}}, \mu_{\delta}\right.$ and $\left.\pi\right)$. The radial velocity was drawn independently. Next, we numerically integrated all clones until their closest approach to the Sun, as in the previous step. The method of how to reconstruct covariance matrix from data in the catalogue is described in Sect. 5.1 of Gaia Collaboration (2016b). Using Cholesky decomposition, we obtained the matrix $(G)$ required for drawing clones, related to the covariance matrix $(C)$ by equation $C=G G^{\prime}$.

In DB15 we noticed that distributions of clones at the minimum distance from the Sun are more or less flat and can be presented in one plane. To find this plane of the maximum scatter of clones we use principal component analysis technique. This approach is described in DB15 (Sect. 2.4), see also Jolliffe (2002).

\section{Results}

\subsection{New close approach parameters of Gliese 710}

After Gaia DR1 was published, Gliese 710 appeared to be the closest flyby star near the Sun and its minimum distance for the nominal solution (i.e. that obtained for original astrometric parameters from the catalogue) is only $13366 \mathrm{AU}$ from the Sun.

\footnotetext{
1 http://simbad.u-strasbg.fr/simbad/
}

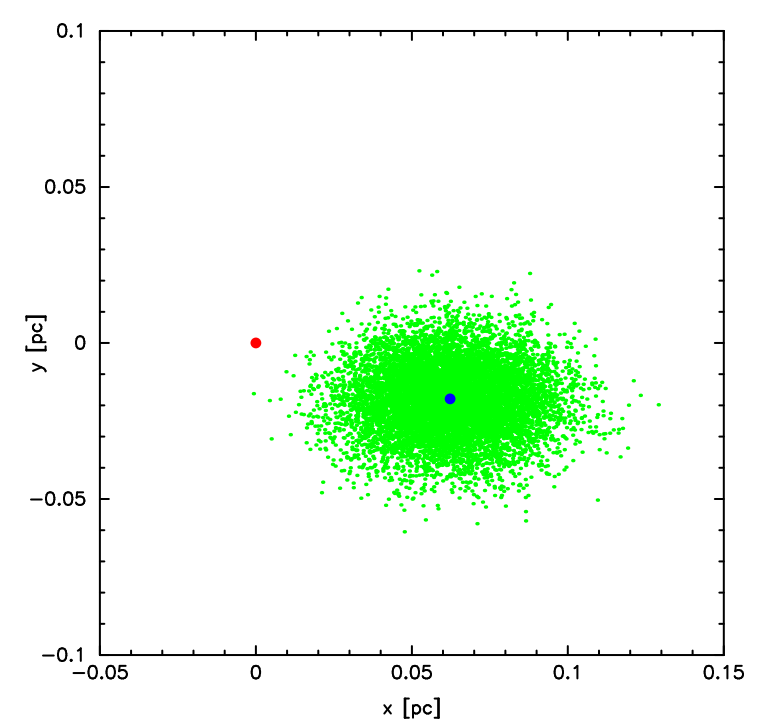

Fig. 1. Distribution of clones of Gliese 710 at the moment of the closest approach to the Sun. See text for a detailed description.

Table 1. New parameters of close approach of Gliese 710.

\begin{tabular}{ccc}
\hline \hline & Mean value & Uncertainty \\
\hline$D[\mathrm{AU}]$ & 13366 & 6250 \\
$T[\mathrm{Myr}]$ & 1.3541 & 0.0500 \\
$V\left[\mathrm{~km} \mathrm{~s}^{-1}\right]$ & 13.7984 & 0.3001 \\
\hline
\end{tabular}

To evaluate the accuracy of this solution we added 10000 clones of this star.

In Table 1 we present new results obtained from the analysis of 10000 clones. We calculate the mean value of minimal distance $D$ from the mean of the heliocentric positions $(\bar{x}, \bar{y}, \bar{z})$. As for the time of passage, the mean value is simply the arithmetic mean, and in both cases the uncertainty is measured as the distance from the mean values containing $90 \%$ of the clones.

The mean proximity distance from the Sun is equal to the nominal solution and $90 \%$ of clones are placed within the radius of $6250 \mathrm{AU}$. Mean time of the closest approach is $T=$ 1.3541 Myr, with an error of 0.0500 Myr and is slightly bigger than the nominal solution.

In Fig. 1 the distribution of clones for the new solution (green dots) is presented. The blue dot marks the position for nominal kinematic parameters and the red dot represents the position of the Sun. The distribution is laid out on the plane of the maximum clone scatter. The principal components analysis (see DB15 for details) was used to determine this plane.

At the moment of its closest approach, Gliese 710 will be one of the brightest objects on the night sky, with an apparent magnitude of $V \simeq-2.742$ (using absolute magnitude value of $M_{V}=8.20$; Koen et al. 2010). Sirius, the brightest star today, has $V \simeq-1.46$; therefore this star will be more than three times brighter than Sirius, and will surpass the brightness of Jupiter and other planets apart from Venus.

Because of the small distance between Gliese 710 and the Sun in the epoch of close approach, the star will become the fastest observed object of the night sky among those outside of the solar system. Total proper motion will be five times greater than the actual proper motion of Barnard Star; it will reach almost one minute of arc per year $(-51.992 \mathrm{arcs} / \mathrm{yr})$ in right accession, and -5.514 arcs/yr in declination. 
Table 2. Compare HIP2 and Gaia DR1 data.

\begin{tabular}{ccc}
\hline \hline & HIP2 & Gaia $\mathrm{DR} 1$ \\
\hline$\alpha$ & $274.96(\mathrm{deg}) \pm 1.48(\mathrm{mas})$ & $274.96(\mathrm{deg}) \pm 0.23(\mathrm{mas})$ \\
$\delta$ & $-1.94(\mathrm{deg}) \pm 1.02(\mathrm{mas})$ & $-1.94(\mathrm{deg}) \pm 0.22(\mathrm{mas})$ \\
$\pi$ & $51.12 \pm 1.63(\mathrm{mas})$ & $52.35 \pm 0.27(\mathrm{mas})$ \\
$\mu_{\alpha *}$ & $1.15 \pm 1.66(\mathrm{mas} / \mathrm{yr})$ & $-0.47 \pm 0.13(\mathrm{mas} / \mathrm{yr})$ \\
$\mu_{\delta}$ & $1.99 \pm 1.22(\mathrm{mas} / \mathrm{yr})$ & $-0.18 \pm 0.09(\mathrm{mas} / \mathrm{yr})$ \\
$R V$ & $13.8 \pm 0.3\left(\mathrm{~km} \mathrm{~s}^{-1}\right)$ \\
\hline
\end{tabular}

\subsection{Comparison of results with previous data}

Gliese 710 is a well-known future visitor of the solar system. In our previous work (DB15), we estimated the minimum distance from the Sun to be $59817 \mathrm{AU}$, with an uncertainty of over $72000 \mathrm{AU}$, while in the work of Bailer-Jones (2014) the obtained minimum distance was 55692 AU with an uncertainty of over 90000 AU. The star's close passage was discussed in many previous papers on this topic, see for example Mullari \& Orlov (1996), Dybczyński \& Kankiewicz (1999), García-Sánchez et al. (2001), Dybczyński (2006). In all these works the star is identified as one of the most important perturbers of the Oort Cloud in a period of ten million years either side of the present day. It has a very small proper motion; in Table 2 we can see that $\mu_{\alpha *}$ is in the range of formal error from HIP2 catalogue, but $\mu_{\delta}$ goes out of this range (for the sake of clarity we did not retain more than two decimal digits, but the differences in $\alpha$ and $\delta$ between the two sources are on the level of mas). The first results from Gaia mission give us the reversed sign in both components and this is the reason why the cloud of clones is on the opposite side of the Sun in respect to that derived from the data from the HIPPARCos mission (see Fig. 2).

Using data from the Gaia mission we obtained results that are ten times more accurate than from HIP2 catalogue, and the closest approaching clone passes the Sun at a distance as close as 2702 AU. $90 \%$ of clones will pass the Sun between 8250 and 19038 AU from the Sun. The estimated most probable minimum distance is more than four times smaller than for nominal solution based on HIP2 data. Figure 2 shows how the diameter of the clone cloud has changed due to Gaia data. The new radius represents less then $10 \%$ of the old one based on HIP2. This shows the precision of Gaia observations. If we look at uncertainties of astrometric parameters, we see that they are 5 to 20 percent of those from HIPPARCos, and we must remember that they are from only one year of Gaia observations.

\subsection{The influence on the Oort Cloud comets}

The encounter with Gliese 710 should not have a significant influence on orbits of major bodies in the solar system, but this star will become a significant perturber for the Oort Cloud comets. The analysis below was performed for the nominal proximity distance of $13365 \mathrm{AU}$, but we stress that the uncertainty is still on the level of $6250 \mathrm{AU}$, and stronger interactions remain possible.

We can extrapolate the expected comet end state probabilities obtained from simulations by Dybczyński (2002, see their Figs. 5 and 6). The impulse strength parameter is $\gamma \simeq 0.04$ in this case. After the passage of Gliese 710, up to 0.1 percent of all Oort Cloud comets might be removed from the cloud, and up to 0.01 percent might be pushed into potentially observable orbits. The percentage of the comets lost from the cloud was fully confirmed in more elaborated simulations Dybczyński (2005), where the simultaneous action of stellar and Galactic perturbations were taken into account.

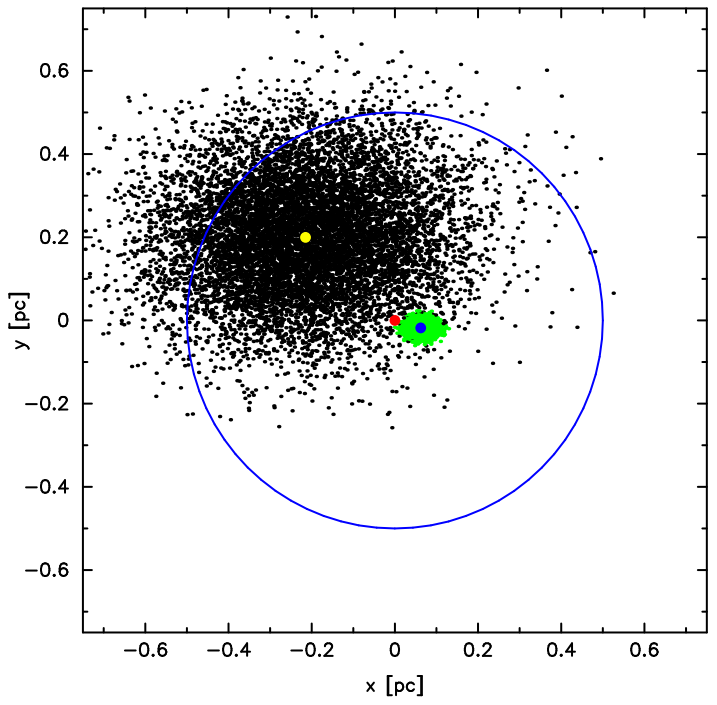

Fig. 2. Comparison of distributions of clones from Gaia and HIPPARCos data, presented on the same plane as in Fig. 1. Black points represent the positions of clones from the HIP2 catalogue, and green dots represent those from Gaia DR1. Yellow and blue dots represent the nominal solutions for HIP2 and Gaia DR1, respectively. A red point shows the position of the Sun. The blue circle depicts the size of the Oort cloud.

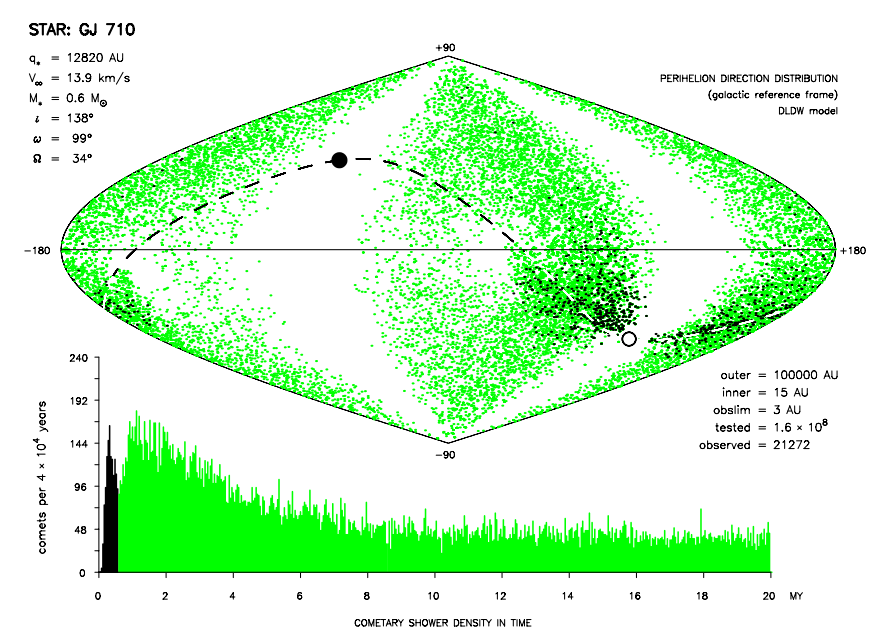

Fig. 3. Structure of the observable comet swarm triggered by Gliese 710. The initial parameters of the star are presented in the upper left corner while the parameters of the simulation are shown in the lower right one. The DLDW model (Dones et al. 1998) of the cloud was used. The projection of the GJ 710 heliocentric orbit is marked by a dashed line. The stellar perihelion (the position at the proximity) is shown as a big, black dot, while the small open circle denotes its antiperihelion direction. The observable comet influx begins shortly after the star passage and gains its maximum 1 Myr later.

The timings and the spatial structure of the swarm of observable comets triggered by the Gliese 710 close passage was presented in Dybczyński (2006; see their Fig. 3), based on the HIPPARCos data and the extended simulations with Galactic, stellar and planetary perturbations taken into account. Here, we repeated those simulations with new encounter parameters, and the results are presented in Fig. 3. The simulation method is fully described in the quoted paper; we only stress here that, before the Gliese 710 passage, we removed all potentially observable comets from the cloud, so none of the simulated observable comets presented in Fig. 3 would be obtained without the gravitational action of the star in question. Figure 3 can be directly compared with Fig. 3 from Dybczyński (2006), except 
for the size of the simulated cometary cloud population, which is 40 times smaller here due to computer time limitations. We present spatial distribution (in Galactic coordinates) of observable comets triggered by Gliese 710 (upper part), as well as the time structure of this cometary shower (lower part). Much closer star passage described in this paper results in an observable comet stream containing 35 times the number of comets in comparison to results derived from HIPPARCOS data (after population rescaling). When we take into account the expected real population of the Oort Cloud $\left(10^{11}\right)$ this means that Gliese 710 will trigger an observable cometary shower with a mean density of approximately ten comets per year, lasting for 3-4 Myr. Moreover, comets arriving during the first 0.6 Myr after the stellar passage (those triggered directly towards the Sun) will be concentrated near the star anti-perihelion direction (black dots and black part of the histogram). The remainder of observable comets will be highly dispersed over large regions of the celestial sphere. In Fig. 3 we show only the first 20 Myr after Gliese 710 passage.

\section{Discussion}

For decades Gliese 710 has been one of the best candidates for a future visitor in the vicinity of the solar system, but before the first Gaia data release its published close approach parameters had large uncertainties. Bailer-Jones (2014) found it to be $90756 \mathrm{AU}$ for $95 \%$ of clones with an average distance of $55691 \mathrm{AU}$. In DB15, this distance amounted to $72193 \mathrm{AU}$ for $90 \%$ of clones with mean position of 59817 AU from the Sun. Thanks to the Gaia mission we can determine the minimum distance with an uncertainty of $6188 \mathrm{AU}$ for the average distance of only 13407 AU from the Sun, which makes this star the closest known future visitor near to the Sun.

Soon after Gaia DR1 was published, the work of Stassun \& Torres 2016 was released. The authors suggested that the parallaxes from Gaia have a systematic offset of -0.25 mas in the sense that they are too small. This conclusion is based on a study of more than one hundred inferred parallaxes in a well-studied binary system. If we include this offset in our calculation, the minimum distance for Gliese 710 decreases to $12788 \mathrm{AU}$ from the Sun and takes place 10000 years earlier.

The $g$ parameter, defined by Feng \& Bailer-Jones (2015), is a kind of measure of the influence of close encounters on Oort Cloud objects. For this star it is equal to $12.09 M_{\odot} \mathrm{pc}^{-2} \mathrm{~km}^{-1} \mathrm{~s}$. This shows that new parameters make this event the strongest disrupting encounter in the future and history of the solar system. The new value is 20 times greater then the old one of $0.60 M_{\odot} \mathrm{pc}^{-2} \mathrm{~km}^{-1} \mathrm{~s}$ from Feng \& Bailer-Jones (2015). In the quoted work, the highest $g$ parameter was found for HIP 85605 $\left(g=3.12 M_{\odot} \mathrm{pc}^{-2} \mathrm{~km}^{-1} \mathrm{~s}\right)$, but this star does not have a credible astrometric solution (DB15, Bailer-Jones 2014). In the TGAS catalogue there is also no data for this object, so for now we can not say anything new in this case.

\section{Conclusion}

Based on the first data from the ESA space mission Gaia we have found new parameters of the close approach of Gliese 710. From our calculations we can expect that this star will have the strongest influence on the Oort Cloud objects in the next ten million years, and even in last several million years there has not been any such important object near the Sun. At minimum distance, this star will be the brightest and the fastest object on the night sky formed outside the solar system. The flyby of Gliese 710 will generate a large flux of new LPCs and many of them will be able to reach the inner part of the solar system.

It should be stressed that such a significant correction of Gliese 710 proximity parameters is mainly due to new proper motions obtained from Gaia results. As stated in Gaia Collaboration (2016a) these new proper motions are highly superior to those from HIPPARCOS or Tycho-2, especially for a single star. This means that proximity parameters presented here are close to reality.

These results additionally show how important the Gaia mission is for the knowledge of the solar neighbourhood. Results based on the first year of work of this spacecraft have significantly improved the accuracy of close stellar approach parameters. We can now expect that after the final data release we will be able to describe the history and future of such phenomena, including also small objects such as brown dwarfs, etc.

Acknowledgements. We are very grateful to the anonymous referee of this paper for valuable remarks. This work has made use of data from the European Space Agency (ESA) mission Gaia (http://www.cosmos.esa.int/gaia), processed by the Gaia Data Processing and Analysis Consortium (DPAC, http://www. cosmos.esa.int/web/gaia/dpac/consortium). Funding for the DPAC has been provided by national institutions, in particular the institutions participating in the Gaia Multilateral Agreement. This research has made use of the SIMBAD data base, operated at CDS, Strasbourg and of NASA's Astrophysics Data System. This research was partially supported by the project 2015/17/B/ST9/01790 founded by the National Science Centre in Poland.

\section{References}

Bailer-Jones, C. A. L. 2014, A\&A, 575, A35

Dones, L., Duncan, M. J., Levison, H. F., \& Weissman, P. R. 1998, in BAAS, 30 1113

Dybczyński, P. A. 2002, A\&A, 396, 283

Dybczyński, P. A. 2005, A\&A, 441, 783

Dybczyński, P. A. 2006, A\&A, 449, 1233

Dybczyński, P. A., \& Berski, F. 2015, MNRAS, 449, 2459

Dybczyński, P. A., \& Kankiewicz, P. 1999, in Evolution and Source Regions of Asteroids and Comets, eds. J. Svoreň, \& E. M. Pittich (Tatranská Lomnica: Astron. Inst. Slovak Acad. Sci.), IAU Colloq., 173, 345

Dybczyński, P. A., \& Królikowska, M. 2015, MNRAS, 448, 588

Feng, F., \& Bailer-Jones, C. 2015, MNRAS, 454, 3267

Gaia Collaboration (Brown, A. G. A., et al.) 2016a, A\&A, in press DOI: $10.1051 / 0004-6361 / 201629512$

Gaia Collaboration (Lindegren, L., et al.) 2016b, A\&A, in press DOI: 10.1051/0004-6361/201628714

García-Sánchez, J., Weissman, P. R., Preston, R. A., et al. 2001, A\&A, 379, 634

Høg, E., Fabricius, C., Makarov, V. V., et al. 2000, A\&A, 355, 27

Irrgang, A., Wilcox, B., Tucker, E., \& Schiefelbein, L. 2013, A\&A, 549, A 137

Jiménez-Torres, J. J., Pichardo, B., Lake, G., \& Throop, H. 2011, MNRAS, 418, 1272

Jolliffe, I. T. 2002, Principal component analysis, 2nd edn. (Springer)

Kaib, N. A., Becker, A. C., Jones, R. L., et al. 2009, ApJ, 695, 268

Koen, C., Kilkenny, D., van Wyk, F., \& Marang, F. 2010, MNRAS, 403, 1949

Michalik, D., Lindegren, L., \& Hobbs, D. 2015, A\&A, 574, A115

Mullari, A. A., \& Orlov, V. V. 1996, Earth Moon Planets, 72, 19

Oort, J. H. 1950, Bull. Astron. Inst. Nether., 11, 91

Stassun, K. G., \& Torres, G. 2016, ApJ, 831, L6

van Leeuwen, F. 2007, A\&A, 474, 653 